\title{
Quality of life in patients with stable disease after surgery, radiotherapy, and chemotherapy for malignant brain tumour
}

\author{
Anna R Giovagnoli
}

\begin{abstract}
Objectives-to evaluate quality of life in patients with malignant brain tumour with stable disease after combined treatments in comparison to patients with other chronic neurological conditions, and to explore the relation of quality of life to clinical, pathological, affective and cognitive factors.

Methods-fifty seven patients who were stable after surgery, radiotherapy and chemotherapy and 24 controls with spastic paraparesis, peripheral neuropathies, myasthenia, ataxia, Parkinson's disease, or multiple sclerosis, were studied. Patients were evaluated by functional living index-cancer, Karnofsky performance status, activity of daily living, self-rating depression scale, state-trait anxiety inventory, and tests for cognitive abilities.

Results-separate Mann-Whitney test comparisons did not show any difference in measures of health related quality of life (functional living index-cancer), autonomy in daily life (activity of daily living), or mood between tumour and control patients, although the first had slower mental speed and worse attention. Seventy three per cent of patients with brain tumour and $58 \%$ of the control patients continued or resumed previous work activity. Quality of life was significantly associated with depression, state anxiety, and performance status in the patients with brain tumour, whereas in control patients, state anxiety was the only factor related to quality of life.

Conclusions-after intensive multimodality treatments, selected patients with brain tumour with stable disease may have satisfactory quality of life that may be not worse than in patients with other chronic neurological illnesses. During the period of stable disease, depressed mood, possibly a reaction to impaired physical and cognitive performance, seems to play a major role in determining quality of life. (F Neurol Neurosurg Psychiatry 1999;67:358-363)
\end{abstract}

Keywords: malignant brain tumour; quality of life

Without further treatment after surgery, patients with high grade glioma survive about three months, ${ }^{1-4} 5$ whereas with intensive treatment patients with anaplastic astrocytoma can survive 36 to 60 months ${ }^{13}$ and patients with glioblastoma survive 10 to 24 months. ${ }^{12}$ How- ever, combined postoperative therapies require repeated admissions to hospital that interfere with patients' home and occupational lives and may also affect the lives of relatives. Furthermore these treatments cause early and late side effects $^{6}$ that require clinical, haematological, neuroradiological, and neuropsychological assessment. Thus the efficacy of postoperative therapies in prolonging survival must be weighed against the possibly deleterious changes in patient lifestyle imposed by these therapies, and this can only be done by assessing the quality of life (QOL) during medium to long term follow up. Self reporting of acceptable or satisfactory QOL after aggressive multimodality treatments can reasonably justify such treatments even in the absence of greatly improved survival.

Unlike patients with other types of tumour (particularly lung, breast, and bowel cancer), ${ }^{7-8}$ patients with brain tumour are rarely assessed for QOL. Studies have assessed QOL in longterm brain cancer survivors ${ }^{9-11}$ and those with variable disease duration, ${ }^{12-15}$ but only a few studies have compared patients at different disease stages. ${ }^{16}$ The psychometric properties (face, constructional, and concurrent validity and reliability) of QOL instruments in patients with brain tumour have recently received more attention. ${ }^{13}{ }^{16-18}$ For example, the functional living index-cancer (FLIC), a self administered visual analogue that explores different dimensions of QOL (physical, emotional, social and occupational aspects, and drug side effects), was investigated in a population of 837 patients with cancer $^{19}$ and was shown to have appropriate content and structure validity, as well as concurrent validity with respect to Karnofsky performance status (KPS) ${ }^{20}$ and the state-trait anxiety inventory (STAI). ${ }^{21}$ In 101 patients with brain tumour, ${ }^{16}$ FLIC showed concurrent validity with respect to STAI, the self rating depression scale (SRDS), ${ }^{22}$ KPS, and attention tests, as well as discriminant validity with respect to disease status and tumour location. Another validated QOL instrument is the brain cancer module (BCM20) ${ }^{17}$; this is a structured questionnaire that explores emotional distress, future uncertainty, visual disorders, motor dysfunction, and communication deficit, using two parallel versions of 20 questions (one for the patient and the other for relatives). In 105 patients with brain tumour, BCM20 showed appropriate internal consistency, test-retest correlation, and discriminant validity for tumour recurrence and KPS score. ${ }^{17}$ There is also the brain module of the functional assess- 
ment of cancer therapy (FACT-Br), ${ }^{18}$ a self administered questionnaire exploring physical, emotional, social, and cognitive dimensions. In 101 patients with brain tumour, FACT-Br had adequate content validity, test-retest correlation, and internal consistency. ${ }^{18}$

Although it seems that tumour recurrence has a major effect in worsening QOL in almost all patients with brain tumour, ${ }^{16}$ the role of other pathological (tumour grading and location), clinical, demographic (age, sex, education, marital status, physical and cognitive performance, and mood) and surgical factors (type and extent of surgical ablation) in determining QOL at different disease epochs is less clear. $^{911131516}$

This study evaluated QOL and its relation to the above factors in patients with malignant brain tumour with stable disease after surgery, radiotherapy, and chemotherapy. The specific aims were $(a)$ to assess QOL in patients with brain tumour compared with patients with other chronic neurological diseases and $(b)$ to explore the relation of QOL to clinical, pathological, affective, and neuropsychological factors in these patients.

\section{Methods}

PATIENTS

Fifty seven patients with malignant brain tumour and stable disease after surgery (biopsy or resection), postoperative radiotherapy, and chemotherapy were recruited. Twenty four inpatients with other chronic neurological diseases served as controls.

Selection criteria for the tumour patients were (a) absence of clinical or neuroradiological signs of disease recurrence (stable disease) and radionecrosis; (b) histological diagnosis; (c) informed consent; and (d) older than 14 years. The control patients (older than 14 years) were recruited during admission to hospital for treatment after informed consent. The patients with brain tumour were a subgroup of 173 patients who received chemotherapy before or after recurrence of disease between January 1995 and June 1997 and who had been referred from surgical departments at our Institute or other Italian hospitals. Sixty two of these patients conformed to the initial selection criteria, but two (3\%) refused to answer the questionnaires considering them not pertinent to their problems, and three others $(4 \%)$ could not answer due severe language difficulties.

The patients with brain tumour knew their diagnoses and that their condition required radiotherapy and chemotherapy. They were also informed that the aim of these postoperative treatments was to slow tumour progression or prevent recurrence. However, they were not informed by staff at this Institute of the prognosis in terms of survival and disability. It cannot be assumed, in all cases, that this information was not obtained elsewhere (from other hospitals); however, none of our patients with brain tumour showed that they knew they had a fatal disease.

The location and type of brain tumour (neuroepithelial tumour, anaplastic astrocytoma, anaplastic oligodendroglioma, and glioblas- toma) varied. The low percentage of glioblastomas $(14 \%)$ is explained by the inclusion criterion of stable disease, which is rarely achieved by these patients. Most patients (82.5\%) underwent surgical resection. Whole brain radiotherapy was given at a dose of 40 Gy plus a $10-15$ Gy boost to the target volume plus 2 $\mathrm{cm}$ margin. The mean interval between radiotherapy and QOL evaluation was 27.02 (SD 28.01) months (range 1-183); in five patients the interval was less than 6 months). A criterion for chemotherapy was that patients should have a KPS score $\geqslant 60$ at the time of therapy planning. All patients with glioma received systemic chemotherapy with carmustine $\left(120 \mathrm{mg} / \mathrm{m}^{2}\right)$ plus cisplatinum $\left(90 \mathrm{mg} / \mathrm{m}^{2}\right)$ for four or five courses at 6 week intervals. Eleven patients with glioma had an Ommaya reservoir fitted during surgery and received locoregional chemotherapy with mitoxantrone ( $3 \mathrm{mg}$ for 3 days every 20 days). Patients with neuroepithelial tumour received four or five courses of systemic chemotherapy with carboplatin $\left(700 \mathrm{mg} / \mathrm{m}^{2}\right)$ plus etoposide $\left(300 \mathrm{mg} / \mathrm{m}^{2}\right)$ every 4 weeks. These patients also received intrathecal chemotherapy with methotrexate $(15 \mathrm{mg})$ plus Ara-C $(60 \mathrm{mg})$ repeated three times with 1 day intervals for three cycles. The mean interval between chemotherapy and QOL evaluation was 24.54 (SD 18.89) months (range 2-85); in four patients the interval was less than 6 months).

The control group consisted of patients with myasthenia $(n=4)$, spastic paraparesis $(n=9)$, adrenoleukodystrophy $(n=1)$, peripheral neuropathy $(n=2)$, multiple sclerosis $(n=6)$, Parkinson's disease $(n=1)$, or cerebellar atrophy $(n=1)$. The selection criteria were presence of significant nervous system disturbances and informed consent. All except the patient with Parkinson's disease received steroid therapy, either before or at the time of the QOL evaluation.

Sex distribution was similar in the two groups. Mann-Whitney test comparison showed that disease duration was longer in the control group $(U=446, p=0.013)$, and patients with brain tumour were younger ( $U=446$, $\mathrm{p}=0.013)$ and had more education $(U=472, \mathrm{p}=$ $0.025)$ than controls. Table 1 summarises the characteristics of both groups of patients.

RATING SCALES FOR QOL

The FLIC was used to measure QOL, ${ }^{19}$ and chosen because of its concurrent and discriminant validity in patients with brain tumour ${ }^{16}$ and its applicability to non-cancer patients. ${ }^{23}$ The FLIC contains 22 questions which cover six QOL dimensions: physical wellbeing (five questions), ability to work (three questions), emotional status (seven questions), sociability (two questions), family situation (three questions), and nausea (two questions). Patients answer the questions by placing a mark on a visual analogue scale divided into seven intervals; each interval corresponds to the response score and the total score is the sum of all responses (maximum score 154); higher scores reflect greater perceived wellbeing. ${ }^{16}{ }^{19}$ 
Table 1 Demographic and clinical characteristics of patients with brain tumour and controls

\begin{tabular}{|c|c|c|}
\hline & $\begin{array}{l}\text { Brain tumour } \\
\text { patients } \\
(n=57)\end{array}$ & $\begin{array}{l}\text { Control } \\
\text { patients } \\
(n=24)\end{array}$ \\
\hline Males & 30 & 11 \\
\hline Females & 27 & 13 \\
\hline \multirow[t]{2}{*}{ Age } & 39.95 & 49.29 \\
\hline & $(11.80)$ & $(16.58)$ \\
\hline Education & $10.09(4.2)$ & $7.75(3.04)$ \\
\hline Married & 40 & 16 \\
\hline Unmarried & 17 & 8 \\
\hline Disease duration (months) & $\begin{array}{c}37.05 \\
(54.36) \\
(3-84)\end{array}$ & $\begin{array}{l}81.42 \\
(86.33) \\
(3-300)\end{array}$ \\
\hline \multicolumn{3}{|l|}{ Tumour type: } \\
\hline Neuroepithelial tumour & 10 & \\
\hline Anaplastic oligodendroglioma & 2 & \\
\hline Anaplastic astrocytoma & 37 & \\
\hline Glioblastoma & 8 & \\
\hline \multicolumn{3}{|l|}{ Tumour location: } \\
\hline Anterior right hemisphere & 15 & \\
\hline Posterior right hemisphere & 9 & \\
\hline Anterior left hemisphere & 11 & \\
\hline Posterior left hemisphere & 8 & \\
\hline Midline & 12 & \\
\hline Subtentorial & 2 & \\
\hline \multicolumn{3}{|l|}{ Surgery: } \\
\hline Biopsy & 10 & \\
\hline Resection & 47 & \\
\hline
\end{tabular}

The KPS, a physician assessed measure, was used only for the patients with brain tumour to evaluate daily and work performance. Part time work was included and daily activities (eating, dressing, bathing, mobility, and sphincter control) were assessed. Scoring was according to the original criteria (range $0-100$ ), with a score of 90 or 100 indicating satisfactory functional status. $^{20}$

The index of independence in activity of daily living $(\mathrm{ADL})^{24}$ was administered to patients and relatives to assess patient autonomy in carrying out basic activities (eating, dressing, bathing, mobility, and sphincter control); high scores (range: 0-18) indicate good autonomy.

For psychological assessment of mood, STAI1 and STAI $2^{21}$ and SRDS ${ }^{22}$ were used. The scores $(20-80)$ are proportional to the level of anxiety or depression; a score equal to or greater than 48 on STAI indicates anxiety; and a score equal to or greater than 50 on SRDS indicates mild to moderate depression. All scales were administered by the same neuropsychologist to all patients.

NEUROPSYCHOLOGICAL TESTS

A preliminary clinical assessment was performed to verify that patients could understand the test procedures, thus excluding those with severely impaired auditory comprehension. Because cognitive abilities are often affected in patients with brain tumour, ${ }^{16}$ a short neuropsychological test battery was included to assess abstract reasoning (Raven's coloured progressive matrices), ${ }^{25}$ attention (attentional matrices), ${ }^{26}$ visual-motor and conceptual tracking (trail making test), ${ }^{27}$ and episodic memory (story recall). ${ }^{28}$

DATA ANALYSIS

To compare the mean scores provided by the self evaluation questionnaires and the neuropsychological tests in the two patient groups, separate Mann-Whitney tests were used, and the effects of age, education, and disease duration on the dependent variables were controlled for by separate regression analyses. Significance was assessed as a p level $<0.005$ (after Bonferroni's correction for 10 measures at an overall error rate of $\alpha<0.05$ for each factor).

In patients with brain tumour, MannWhitney tests were used to compare FLIC scores with demographic factors (sex, marital status) and surgical procedure (biopsy $v$ resection). Kruskall-Wallis one way analysis of variance (ANOVA) was used to compare patients on the basis of pathological factors (histological type, tumour location).

Pearson's coefficients and multiple stepwise regression analysis were performed to explore the association of FLIC scores with KPS, STAI, SRDS, ADL, neuropsychological test scores, age, education, and duration of disease.

\section{Results}

Table 2 shows the mean scores, SD, and ranges for the scales and neuropsychological tests in the brain tumour and control groups.

COMPARISONS BETWEEN PATIENTS WITH BRAIN TUMOUR AND CONTROLS

Patients in the brain tumour group had a KPS score in the range 60 to 100 , and $73 \%$ of them had resumed the job (including homemaker and part time work) they had ceased at the time of diagnosis. Fourteen control patients had impaired physical autonomy and sometimes needed a wheelchair or walking stick. Fourteen control patients $(58 \%)$ resumed previous work (including part time work).

As required by the selection criteria, no patients had aphasic disorders of severity such as to impede test administration or form compilation. To control for deficits in abstract reasoning, individual scores on Raven's coloured progressive matrices were adjusted for age and education. Four patients with brain tumour had an adjusted score of 18 that is the cut off for normal and pathological performance, and one patient obtained an adjusted score of 12

Table 2 Mean scores (SD) and ranges for the self administered scales and neuropsychological tests in brain tumour and control patient groups

\begin{tabular}{lcc}
\hline & $\begin{array}{c}\text { Brain tumour } \\
\text { patients }\end{array}$ & Control patients \\
\hline FLIC & $116.63(21.37)$ & $105.46(19.5)$ \\
& $67-154$ & $60-137$ \\
ADL & $17.07(1.82)$ & $17.21(1.56)$ \\
& $10-18$ & $12-18$ \\
KPS & $85.96(12.66)$ & \\
& $60-100$ & \\
STAI-1 & $41.35(12.66)$ & $47.08(12.09)$ \\
& $20-78$ & $29-68$ \\
STAI-2 & $42.16(11.47)$ & $42.71(10.67)$ \\
& $20-73$ & $17-61$ \\
SDRS & $39.28(10.06)$ & $37.42(7.66)$ \\
& $24-60$ & $24-51$ \\
Progressive matrices & $27.86(6.37)$ & $26.0(5.22)$ \\
& $7-36$ & $17-35$ \\
Attentional matrices & $46.81(12.74)$ & $50.79(6.21)$ \\
& $15-60$ & $40-59$ \\
Trail making test part A & $87.28(74.05)$ & $75.75(39.83)$ \\
& $25-420$ & $30-150$ \\
Trail making test part B & $232.35(183.7)$ & $185.0(113.17)$ \\
& $60-995$ & $60-608$ \\
Story recall & $9.94(4.97)$ & $10.65(4.75)$ \\
& $1.5-23.5$ & $2-22.5$ \\
\hline
\end{tabular}


(pathological) but this was due to perceptive difficulties; all the other patients with brain tumour had normal scores.

The patients with tumour had better FLIC scores and worse trail making test (part B) scores than controls. However, separate MannWhitney tests did not show any differences between the tumour and control groups in terms of score for FLIC $(U=476.5, \mathrm{p}=0.031)$, ADL $(U=674, \quad \mathrm{p}=0.89), \quad$ STAI1 $(U=502$, $\mathrm{p}=0.059)$, STAI2 $(U=641, \mathrm{p}=0.65)$, SRDS ( $U=618, p=0.49$ ), Raven's coloured progressive matrices $(\mathrm{U}=533, \mathrm{p}=0.11)$, attentional matrices $(U=624, \mathrm{p}=0.53)$, trail making test part A $(U=673.5, \mathrm{p}=0.91)$ and $\mathrm{B}(U=624$, $\mathrm{p}=0.53)$, or story recall scores $(U=637$, $\mathrm{p}=0.62$ ). As assessed by separate multiple stepwise regression analyses, the level of education had effects on the scores of STAI2 $(p=0.03)$, Raven's coloured progressive matrices $(p=0.0001)$, trail making test part A $(p=0.02)$ and $B(p=0.001)$, and story recall $(p=0.01)$. Age had a significant effect on Raven's coloured progressive matrices $(\mathrm{p}=0.008)$, whereas disease duration had no effect on any score.

RELATION OF FLIC SCORE TO PATHOLOGICAL, CLINICAL AND NEUROPSYCHOLOGICAL FACTORS Separate Kruskall-Wallis one way ANOVAs comparing patients with brain tumour on the basis of tumour location (anterior right 112.66 (SD 23.68); posterior right 115.88 (SD22.89); anterior left 118 (SD 16.36); posterior left 109.25 (SD 19.95); midline structures 120.25 (SD 21.26); subtentorial 150 (SD 0) $\left(\chi^{2}=6.75\right.$, $\mathrm{p}=0.23)$ ), and tumour type (neuroepithelial tumour 126.2 (SD 23.15); anaplastic oligodendroglioma 110 (SD 4.24); anaplastic astrocytomas 113.13 (SD 22.07); glioblastomas 122.5 $\left.(\mathrm{SD} 14.41)\left(\chi^{2}=3.73, \mathrm{p}=0.29\right)\right)$ did not show any differences. Separate Mann-Whitney tests did not show any differences in FLIC scores for sex (males 114.73 (SD 21.08), females 118.74 (SD 21.88) $(U=365.5, \mathrm{p}=0.52))$, marital status (married: 117.92 (SD 21.73), unmarried 113.58 (SD 20.79) ( $U=289.5, \mathrm{p}=0.37)$ ), or surgical procedure (biopsy 119.50 (SD 21.97), resection 116.02 (SD 21.42) ( $U=225$, $\mathrm{p}=0.83)$ ).

In patients with brain tumour, Pearson's coefficient did not show any correlation of FLIC score with age, education or disease duration. Significant correlations were found between FLIC score and the scores for STAI1 $(r=-0.57, \quad \mathrm{p}<0.001), \quad \mathrm{STAI} 2 \quad(r=-0.63$, $\mathrm{p}<0.001)$, SRDS $(r=-0.75, \mathrm{p}<0.001), \operatorname{KPS}(r=$ $0.40, \mathrm{p}<0.001$ ), trail making test (part A $r=-0.39, \mathrm{p}<0.01$; part B: $r=-0.38, \mathrm{p}<0.01)$ and attentional matrices $(r=0.36, \mathrm{p}<0.01)$. Multiple stepwise regression analysis, taking the factors related to FLIC score as independent variables, showed significant associations of FLIC score with SRDS $(F=70.36, \mathrm{p}<0.0001)$, KPS $(F=39.50, \mathrm{p}<0.0001)$, and STAI1 scores $(F=30.70, \mathrm{p}<0.0001)$.

In control patients, Pearson's coefficient did not show any correlation between FLIC score and age, education, disease duration, or scores from the self evaluation and neuropsychologi- cal tests. Multiple stepwise regression analysis with the same independent factors used in patients with brain tumour showed that FLIC score was associated with STAI1 score $(F=5.04, \mathrm{p}=0.035)$.

\section{Discussion}

This study explored QOL in a highly selected sample of patients with brain tumour who were well enough to receive combined postoperative radiotherapy and chemotherapy and were stable thereafter. The control group was a heterogeneous group of patients with chronic disabling disorders of the central or peripheral nervous system.

Mean FLIC score, a health related QOL measure, was 116 in our patients with brain tumour. This is higher than the 107 reported by Schipper et $a l^{19}$ in a general cancer population, and the 107 reported in patients with lung cancer. ${ }^{8}{ }^{29}$ This suggests that aggressive combination treatment of patients with brain tumours does not necessarily affect QOL more than therapies used for cancer not involving the nervous system, although brain radiotherapy and chemotherapy may have late effects on cognitive abilities and daily performance. ${ }^{61130}$ The results of this study further indicate that QOL, as expressed by FLIC and instruments assessing specific dimensions of QOL (mood, daily performance, autonomy), is not worse in patients with brain tumour than in patients with other chronic neurological diseases. This may be in part because the patients with brain tumour were chosen for chemotherapy on the basis of good performance status (KPS $\geqslant 60$ ) which could be a prognostic factor for good QOL after treatment. Furthermore, as required by the selection criterion of stable disease, these patients did not have seriously disabling motor or cognitive impairment, and only $14 \%$ had glioblastoma. Nevertheless, it is likely that these results can be generalised to patients with stable disease, including patients with glioblastoma, as status of disease, and not histological type, is the main influence on QOL after combined treatments. ${ }^{16}$ However, the present patient sample is not representative of patients with brain tumour in general, because it specifically excluded untreated patients and those with recurrence of disease.

It is noteworthy that $73 \%$ of patients of this tumour series resumed their previous occupations, compared with $58 \%$ of control patients, even though the level of autonomy in everyday activities (as expressed by ADL) was similar in the two patient groups. This suggests that the ability to work (closely related to the perception of satisfactory QOL) is not compromised by combination treatments in all patients with brain tumour and is in accord with the findings of Kleinberg et $a l^{31}$ who reported that $68 \%$ of patients with stable disease returned to work after surgery and radiotherapy, $62 \%$ of whom were at work 1 year later, and $58 \%$ of whom were still working after 2 to 4 years.

None of the patients with brain tumour in this study were demented or had disabling comprehension difficulties, thus the answers 
they gave in the self evaluation questionnaires are probably reliable and provide a real measure of QOL. Mental slowing was evident and related to the level of QOL, indicating that compromise of attention may play a part in determining wellbeing in these patients.

Patients with brain tumour and controls did not differ in self reporting of depression or anxiety. However, in the brain tumour group depression and state anxiety were closely associated with QOL, whereas in the control group QOL was associated only with state anxiety, suggesting that different factors are modulating mood in the two groups. Although the patients with brain tumour knew their diagnoses, a detailed assessment of what each knew about the prognosis was not carried out. In no case was survival or dying openly mentioned by these patients. Families tried to protect patients against the truth, and it is the policy of medical staff never to remove hope. The overall impression was that the QOL found in these patients represented that of patients who knew they had a serious disease, but that the prognosis was not hopeless. We therefore conclude that depressed mood in these patients with brain tumour, the main predictor of QOL, is a consequence of the disruption of everyday life and future plans brought about by the extended therapies; perhaps it is also a reaction to the physical and cognitive impairment caused by the tumour and its treatment, but is not due to awareness that death is close. As also suggested by Davies $e t a l,{ }^{32}$ the fact that a specific prognosis was not given together with the arduous and extended treatments, is likely to have made the patients feel cared for and to have instilled hope that something could be done.

The lack of association between QOL and depression in the control patients may be a consequence of the fact that they were evaluated in a late stage of the disease, when the initial affective reaction had been overcome and they had adapted to the changes imposed on their lives by chronic illness. The relation of QOL to state anxiety found in both patient groups could be due to anxiety about the evaluation itself or to the admissions to hospital.

In agreement with previous studies on patients with brain tumour, ${ }^{9} 1315$ the present study found no relation between QOL and the histological type of the tumour. This is probably because clinical status is more important than intrinsic tumour malignancy in determining QOL and the patients were homogeneous for the first. Similarly the lack of association between QOL and location of tumour may be attributed to similarity of disease status, thus explaining the difference between this and our previous finding of better QOL in patients with hemispheric anterior right or diencephalic tumour. ${ }^{16}$

In conclusion, the results of this study indicate that QOL can be satisfactory in many patients with brain tumour with stable disease after the end of combination treatments, and is not necessarily worse than in patients with other chronic neurological diseases. Mood, physical performance, and mental speed are major predictors of QOL in patients with stable brain tumour. Long term assessment of QOL may be useful to compare patients who are stable after basic treatments with those undergoing repeated chemotherapy, to weigh the effects of aggressive therapy. Structured assessment of patients' beliefs about their prognosis of survival and disability, could help to understand QOL self reporting more fully.

We thank Dr A Boiardi for providing the opportunity to study the patients with brain tumour and for his constructive criticism of an early version of the manuscript. Thanks are also due to $\mathrm{Dr}$ AL Pozzi and Dr A Silvani for providing treatment data, Dr F Burgio for editorial assistance, and DC Ward for help with the English.

1 Prados MD, Scott CB, Rotman M, et al. Influence of bromodeoxyuridine radiosensitization on malignant glioma patient survival: a retrospective comparison of survival from the Northern California Oncology Group (NCOG) and Radiation Therapy Oncology Group trials (RTOG) for glioblastoma multiforme and anaplastic astrocytoma. Int $\mathcal{F}$ Radiat Oncol Biol Phys 1998;40:653-9.

2 Sneed PK, Stauffer PR, McDermott MW, et al. Survival benefit of hyperthermia in a prospective randomized trial of brachytherapy boost \pm hyperthermia for glioblastoma multiforme. Int f Rad Oncol Biol Phys 1998;40:287-95.

3 Urtasun RC, Kinsella TJ, Farnan N, et al. Survival improvement in anaplastic astrocytoma, combining external radiation with halogenated pyrimidines: final report of RTOG 86-12, Phase I-II study. Int $\mathcal{F}$ Radiat Oncol Biol Phys 1996; 36:1163-7.

4 Walker MD, Alexander E, Hunt WE, et al. Evaluation of $\mathrm{BCNU}$ and/or radiotherapy in the treatment of anaplastic gliomas. A cooperative clinical trial. $\mathcal{F}$ Neurosurg 1978;49: 333-43.

5 Kristiansen K, Hagen S, Kollevold T, et al. Combined modality therapy of operated astrocytomas grades III and IV Confirmation of operated astrocytomas grades III and IV. Confirmation of the value of postoperative irradiation prospective multicenter trial of the Scandinavian Glioblastoma Study Group. Cancer 1981;47:649-52.

6 Duffner PK. Long term effects of treatment. In: Bradly WG, Duffner PK. Long term effects of treatment. In: Bradly WG,
Daroff RB, Fenichel GM, et al, eds. Neurology in clinical practice. London: Butterworth-Heinemann, 1989:1046-7.

7 Yates JW, Chalmer B, McKegney FP. Evaluation of patients with advanced cancer using the Karnofsky performance status. Cancer 1980;40:2220-4.

8 Ganz PA, Haskell CM, Figlin R, et al. Estimating the quality of life in a clinical trial of patients with metastatic lung cancer using the Karnofsky performance status and the functional living index-cancer. Cancer 1988;61:849-56.

9 Hochberg FH, Slotnick B. Neuropsychological impairment in astrocytoma survivors. Neurology 1980;30:172-7.

10 Imperato JP, Palaleogos NA, Vich NA. Effects of treatment on long-term survivors with malignant astrocytomas. Ann Neurol 1990;28:818-22.

11 Giovagnoli AR, Boiardi A. Cognitive impairment and quality of life in long-term survivors with malignant brain ity of life in long-term survivors with

12 Ammirati M, Vick N, Liao Y, et al. Effect of the extent of surgical resection on survival and quality of life in patients with supratentorial glioblastoma and anaplastic astrocytomas. Neurosurgery 1987;21:201-6.

13 Mackworth N, Fobair P, Prados MD. Quality of life self-reports from 200 brain tumour patients: comparisons with Karnofsky performance scores. $\mathcal{F}$ Clin Oncol 1992;14: 243-53.

14 Taphoorn MJB, Heimans JJ, Snoeck FJ, et al. Assessment of quality of life in patients treated for low-grade glioma: a preliminary report. $\mathcal{F}$ Neurol Neurosurg Psychiatry 1992;55: 372-6.

15 Marie J, Coudin B, Guerin J, et al. Neuropsychological impairment in adults with brain tumours. Am $\mathcal{F}$ Clin Oncol 1987;10:156-62.

16 Giovagnoli AR, Tamburini M, Boiardi A. Quality of life in brain tumour patients. F Neurooncol 1996;30:71-80.

17 Osoba D, Aaronson NK, Muller M, et al. The development and psychometric validation of a brain cancer quality-of-life questionnaire for use in combination with general cancerspecific questionnaire. Qual Life Res 1996;6:139-50.

18 Weitzner MA, Meyers CA, Gelke CK, et al. The functional assessment of cancer therapy (FACT) scale: development of a brain subscale and revalidation of the FACT-G in the brain tumor population. Cancer 1995;75:1151-61.

19 Schipper H, Clinch J, McMurray A, et al. Measuring the quality of life of cancer patients: the functional living index-cancer: development and validation. $\mathcal{F}$ Clin Oncol $1984 ; 2: 472-83$

20 Karnofsky DA, Burchenal JH. The clinical evaluation of chemotherapeutic agents in cancer. In: McLoad CM, ed. Evaluation of chemotherapeutic agents. New York: Columbia Evaluation of chemotherapeutic ag

21 Spielberger CD. Inventario per l'ansia di stato e di tratto. Spielberger CD. Inventario per lansia di stato

22 Zung WWK. A self-rating depression scale. Arch Gen Psychiatry 1965;12:63-70. 
23 Tate DG, Johnson-Green L, Forchheimer M, et al. Ouality of life outcome measures in cancer: application in rehabilitation. Ann Arbor: Department of Physical Medicine and Rehabilitation, University of Michigan Medical Center, 1997.

24 Katz S, Downs TD, Cash HR. Progress in development of an index of ADL. Gerontologist 1970;10:20-30.

25 Basso A, Capitani E, Laiacona M. Raven's coloured progressive matrices: normative values on 305 adult normal controls. Funct Neurol 1987;2:189-94.

26 Spinnler H, Tognoni G. Standardizzazione e taratura italiana di test neuropsicologici. Ital f Neurol Sci 1987; suppl 8:47-50.

27 Lezak M. Neuropsychological assessment. New York: Oxford University Press 1995.
28 Novelli G, Papagno C, Capitani E, et al. Tre test clinici di memoria verbale a lungo termine. Taratura su soggetti normali. Archivo di Psicologica Neurologica e Psichiatria

29 Ganz PA, Lee JJ, Siau J. Quality of life assessment. An independent prognostic variable for survival in lung cancer. Cancer 1991;67:3131-5.

30 Newton C, Mateo MA. Uncertainty: strategy for patients with brain tumour and their family. Cancer Nurs 1994;17: 137-40.

31 Kleinberg L, Wallner K, Malkin MG. Good performance status of long-term disease-free survivors of intra-cranial gliomas. Int f Radiat Oncol Biol Phys 1993;26:129-33.

32 Davies E, Clarke C, Hopkins A. Malignant cerebral glioma. II: Perspectives of patients and relatives on the value of radiotherapy. BMf 1996;313:1512-16. 